\title{
Multi-objective Optimization of Compliant Parallel Platform for Desired Stiffness and Workspace
}

\author{
Hongwei Xu, Haibo Zhou*, Zhiqiang Li, Xia Ju. \\ State Key Laboratory of High Performance Complex Manufacturing, Central \\ South University, Changsha 410083, China.
}

* Corresponding author. E-mail: zhouhaibo@csu.edu.cn.

\begin{abstract}
Stiffness and workspace are crucial performance indexes of a precision mechanism. In this paper, an optimization method is presented, for a compliant parallel platform to achieve desired stiffness and workspace. First, a numerical model is proposed to reveal the relationship between structural parameters, desired stiffness and workspace of the compliant parallel platform. Then, the influence of the various parameters on stiffness and workspace of the platform is analyzed. Based on Gaussian distribution, the multi-objective optimization problem is transformed into a single-objective one, in order to guarantee convergence precision. Furthermore, particle swarm optimization is used to optimize the structural parameters of the platform, which significantly improve its stiffness and workspace. Last, the effectiveness of the proposed numerical model is verified by finite element analysis and experiment.
\end{abstract}

Keywords: Compliant parallel platform, Multi-objective optimization, Numerical model, Particle swarm optimization

\section{Introduction}

With parallel structure as its basic configuration, the Compliant Parallel Platform (CPP) has special large stroke compliant joints. Compared to conventional serial platforms, the parallel platforms have higher robustness, bearing capacity, and accuracy [1]. In addition, compliant joints are used to overcome the friction, backlash, and crawling of the conventional mechanism joints [2-5]. CPP can achieve the expected motion via the elastic deformation of its compliant joints. The advantage of parallel structure and compliant joints assures high precision and multiple Degrees Of Freedom (DOF) of CPP. Recently, these platforms have been the focus of many researchers, producing achievements that have been applied in precision machining robotics and other fields.

Stiffness, precision, and workspace $[6,7]$ are typical performance indexes of a mechanism. Though many studies have shown that the precision of CPP is able to 
meet the needs, in most application scenarios, the limited stiffness and workspace of CPPs still confine the scope of their application. In the case of CPP with compliant joints, the effect of structural parameters on stiffness is contrary to their effect on the workspace. Thus, solving the contradiction between high stiffness and wide workspace, in the field of CPPs, has attracted high research interest.

In order to achieve the desired stiffness and workspace $[8,9]$, the structural parameters of CPP ought to be optimized. Meeting the needs in different application scenarios may set a single or multiple objectives to be optimized. In [10], the maximum workspace of the robot is obtained according to constraints imposed by the surrounding settings. In [11], the topology optimization is proposed to improve the global stiffness. In [12, 13], typical non-dimensional performance indices were used to optimize the geometric parameters of a 3-DOF parallel kinematic structure. Considering the performance criteria of the 6-DOF parallel manipulators, a multi-objective optimization is formulated in [7, 14, 15].

Previous optimization approaches in parallel platforms mainly focus on the platform with conventional mechanism joints, while the optimization of CPP is mainly about a single part, a single design objective, or a single structural parameter. Previous research work seldom studied the multi-objective optimization of CPP. In addition, due to the wide use of Finite Element Analysis (FEA), in the investigation of the mechanism stiffness and workspace, the specific parameters in question are discrete. Hence, the mechanism needs to be modeled repeatedly.

In this paper, a numerical optimization for CPP is presented, to achieve desired stiffness and workspace. The target of controllable convergence precision is achieved using a method based on Gaussian distribution which transforms multiobjective optimization problem into a single-objective one. In addition, Particle Swarm Optimization (PSO) is used to optimize the structural parameters of the platform. Moreover, FEA and experiment are used, to compare the stiffness and workspace of the pre-optimization and post-optimization platform, as well as to verify the effectiveness of the proposed numerical optimization. The rest of this paper is organized as follows: Section II describes the system of CPP. Section III presents the Numerical Analysis (NA). Sections IV and V present the parameter optimization and the evaluation of stiffness and workspace, respectively. Last, the conclusion is presented in Section VI.

\section{System description}

The structure of the 6-DOF CPP, as applied in the field of optoelectronic packaging, is shown in Fig. 1. Specifically, the lower fixture of a laser diode and the upper fixture of an optical fiber is fixed on the CPP and the auxiliary arm, respectively. The CPP and the auxiliary arm are fixed on the optical table. This setup enables the laser diode to adjust its posture according to the precise movement of the CPP, in order to achieve alignment between the laser diode and the optical fiber. 


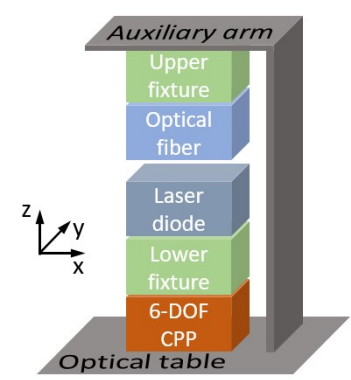

Fig. 1 Structure diagram of an optoelectronic packaging platform.

As is shown in Fig. 2(a), CPP consists of a moving platform, six branch chains, six actuation units, and a fixed platform. At the initial state, the moving and fixed platforms are parallel to the horizontal plane. As is shown in Fig. 2(b), the branch chain consists of two wide-range compliant joints and a rigid rod. The joints connected to the moving platform and the actuation unit are called the upper-joint and the lower-joint, respectively. The origin of coordinates systems B-xyz and Pxyz is located at the center of the circles, as they are defined by the lower-joints and by the upper-joints, respectively. The motion direction of actuation units 1,3 , 4 , and 6 is parallel to $\mathrm{x}$-axis, and the motion direction of actuation units 2 and 5 lies along y-axis. The direction of $\mathrm{z}$-axis of the two coordinates systems is shown in Fig. 2(a). The structure of the platform, prior to optimization, is determined by the eight parameters listed in Table 1.

In this case, the wide-range compliant joints are made of beryllium bronze (QBe2), while other structural parts are made of hard aluminum alloy (2A12). The material attribute parameters are listed in Table 2.

Previous research has achieved satisfying precision, stiffness, and workspace for the platform in the packaging of coaxial optoelectronic device. The need for packaging a wider variety of optoelectronic devices demands higher stiffness and wider workspace of the platform. To this end, a numerical optimization for CPP, to achieve the desired stiffness and workspace, is presented.

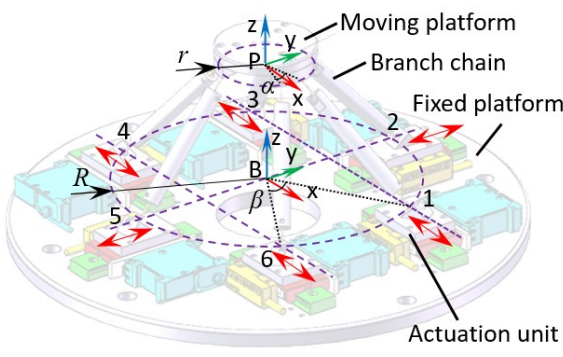

(a)

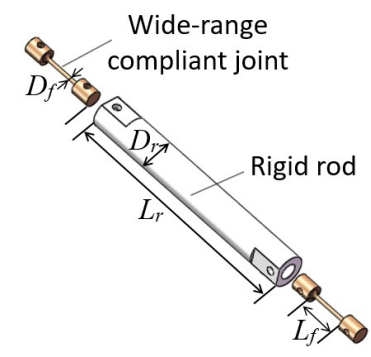

(b)

Fig. 2 Schematic diagram of CPP structure and branch chain.

Table 1 Parameters of the pre-optimized platform.

\begin{tabular}{ccc}
\hline Quantity & Symbol & Value \\
\hline Radius of the moving platform $(\mathrm{mm})$ & $r$ & 25 \\
Radius of the fixed platform (mm) & $R$ & 90 \\
Distribution angle of the upper-joint (deg) & $\alpha$ & 30 \\
Distribution angle of the lower-joint (deg) & $\beta$ & 60 \\
Length of the joint (mm) & $L_{f}$ & 13 \\
Diameter of the joint (mm) & $D_{f}$ & 1 \\
Length of the rigid rod (mm) & $L_{r}$ & 74 \\
Diameter of the rigid rod (mm) & $D_{r}$ & 8 \\
\hline
\end{tabular}


Table 2 Material attribute parameters.

\begin{tabular}{ccc}
\hline Attribute & QBe2 & $2 \mathrm{Al} 2$ \\
\hline Modulus of elasticity $(\mathrm{GPa})$ & 130 & 70 \\
Density $\left(\mathrm{kg} / \mathrm{m}^{3}\right)$ & 8100 & 2700 \\
Yield stress $(\mathrm{MPa})$ & 1035 & 255 \\
Poisson's ratio & 0.35 & 0.33 \\
\hline
\end{tabular}

The mapping between the structural parameters and the desired stiffness and workspace is illustrated in Fig. 3. The static stiffness of the platform is defined as the force required, to make a unit change at the end of the platform. Specifically, when a unit change is made at the end of the platform, along a certain direction, the respective static stiffness of the platform in this direction can be derived by calculating the resultant force of each branch chain. Regarding the establishment of the platform workspace, it is essential to obtain the motion range of every motion pair. Also, considering that the stress of a compliant joint should be limited to values lower than its allowable stress level, the calculated stress value of every joint can perform as a real-time criterion of whether the end of the platform lies within its workspace. In addition, in the process of solving the workspace, leading the end of the platform to move to a certain posture, requires the inverse kinematics model of the platform, in order to derive the motion of every driving component. Therefore, NA process is divided into three parts: the Inverse Kinematics Model (IKM), the workspace model, and the stiffness model.

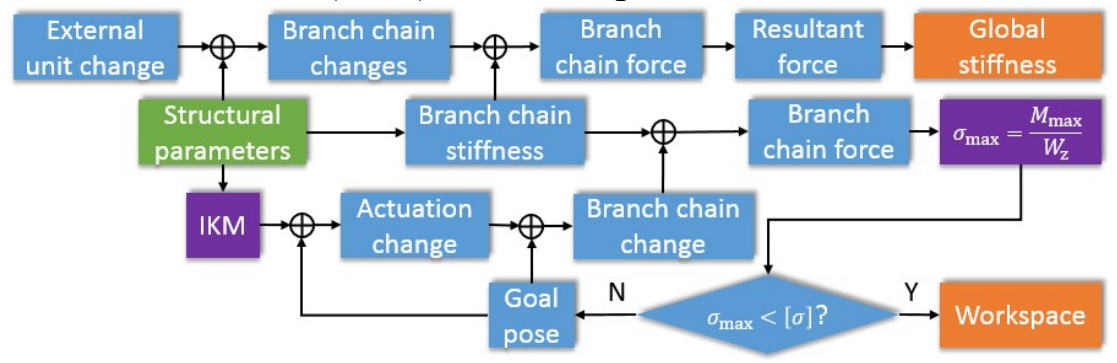

Fig. 3 The mapping between the structural parameters, the global stiffness and the workspace.

\section{Numerical analysis}

\subsection{Inverse kinematics model}

The relationship between loads and displacements in the elastic deformation region can be assumed as linear. The components $\Delta$ of the branch chain $i$ in the local coordinates system are described as:

$$
D_{\Delta \mathrm{i}}=C_{\Delta} F_{\Delta \mathrm{i}}
$$

where, $D=\left[\begin{array}{llllll}d_{x} & d_{y} & d_{z} & \theta_{x} & \theta_{y} & \theta_{z}\end{array}\right]^{\mathrm{T}}$ represents the nodal posture changes, $F=\left[\begin{array}{ll}f_{x} & f_{y} f_{z}\end{array}\right.$ $\left.\begin{array}{lll}M_{x} & M_{y} & M_{z}\end{array}\right]^{\mathrm{T}}$ represents the nodal loads and $C$ is the flexibility matrix of the parts, which can be obtained according to [16]. In Eq. (1), the subscripts $\Delta=\mathrm{a}, \mathrm{b}, \mathrm{c}, \mathrm{d}$ represent the upper compliant joint, rigid rod, lower compliant joint, and actuation units, respectively, while $i=1,2,3,4,5,6$ represent the serial numbers of the branch chain.

The differential nodal forces and displacements of each moving part can be transformed from one coordinates system $M$ to another coordinates system $N$, 
using the equivalent transformation matrix $T_{M}^{N}$. According to the relationship between force and displacement, the inverse kinematics model is derived, as presented in the work [17],

$$
D_{\Delta \mathrm{i}}=C_{\Delta} T_{\mathrm{Pi}}^{\Delta} K_{\mathrm{i}} T_{\mathrm{P}}^{\mathrm{Pi}} D_{\mathrm{P}}
$$

Where, $K_{\mathrm{i}}$ represents the stiffness matrix of the branch chain and the displacement of the actuation units $D_{\mathrm{di}}$ is derived, based on desired posture changes $D_{\mathrm{P}}$.

\subsection{Workspace model}

Without considering the tilt, the workspace is defined by a set of points that can be reached by the center of the moving platform. In order to determine whether a point in space lies within the workspace of the platform, it is necessary to analyze the stress of each part of the platform, when the end of the platform moves to that point. Fig. 4 shows the stress analysis of a branch chain, where its initial state is represented by the black dotted line. According to the structure parameters of the platform, the posture of the upper end $(a)$ and the lower end $(d)$ of the branch chain, in the global coordinates system, can be obtained. When the platform moves to a target position $P^{\prime}$, its posture change is $D_{o}$ and the branch chain lies within the green shaded area (Fig. 4). According to the inverse solution model of the platform, the posture of the lower end of the branch chain in the global coordinates system, represented by $d^{\prime}$, can be obtained. As the deformation of the moving platform is negligible, compared to that of the compliant joint, the posture change of the upper end of the branch chain is equal to the position change of the moving platform. Thus, the posture of the upper end of the branch chain, in the global coordinates system, represented by $a^{\prime}$, can be obtained.

For reasons of simplification, in the stress analysis process of a branched chain, it is assumed that there is no moving platform at its top. In this way, the parts of the branch chain, as illustrated by the red dotted line (Fig. 4), will not deform. According to the structural parameters of the branch chain, the posture of the upper end of the branch chain in the global coordinates system, represented by $a^{\prime \prime}$, can be obtained. The moving platform is substituted by an equivalent force $F_{e q}$, while the force $F_{e q}$ lets the posture of the upper end of the branch chain change from $a^{\prime \prime}$ to $a^{\prime}$. The posture change, denoted by $D_{e q}$, is obtained by the posture of $a^{\prime \prime}$ and $a^{\prime}$, in the global coordinates system. It should be noted that, $D_{e q} \neq D_{o}$. Thus, according to Eq. (1) and the flexibility matrix of the branch chain $C_{b}$, the force $F_{e q}$ is obtained. Then, force synthesis provides the equivalent moment $M_{\max }$. The maximum stress of the branch chain can be obtained by $\sigma_{\max }=M_{\max } / W_{z}$, where $W_{\mathrm{z}}$ is the section modulus in bending of the compliant joint. Next, the maximum stress of the platform, at the target position, is obtained.

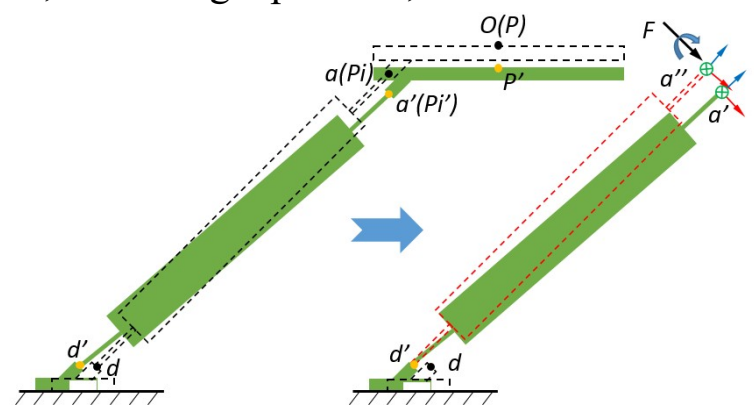

Fig. 4 The stress deformation diagram of the branch chain. 
The compliant joint is the part to be considered, as it is the weakest part of the whole platform. The yield strength of the compliant joint is $1035 \mathrm{MPa}$. In practice, the platform rarely works under the maximum levels of working conditions. Considering the safety factor of 1.25 , the allowable stress $\left[\sigma_{\max }\right]$ is equal to 828 $\mathrm{MPa}$. Thus, the workspace of the platform is determined by the target points, where the maximum stress is less than $828 \mathrm{MPa}$.

\subsection{Stiffness model}

In the initial state, the lower end of the branch chain is fixed. Assuming that an external force $F_{e}$ is applied on the moving platform, resulting in a unit posture change $D_{e}$, similarly to the solution process for the workspace, it is derived that the posture change of the upper end of the branch chain, in relation to its lower end, equals the unit posture change $D_{a}$. Based on Eq. (2) and the flexibility matrix $C_{r}$ of the branch chain, the equivalent force $F_{r}$ of the end of the branch chain is obtained. According to the balance principle of force, the resultant force of all branch chains and the external force $F_{e}$ are a pair of balanced forces. Therefore, by decomposing the equivalent force of all branch chains into the direction of the external force and composing the produced forces, the magnitude of the external force is obtained. The static stiffness of the platform can be obtained by $K_{e}=F_{e} /$ $D_{e}$.

\section{Parameter optimization}

\subsection{Parameter analysis}

Based on the pre-optimized platform parameters, the above mathematical model is used to analyze the influence of the various parameters on the travel and stiffness of the platform, in the $\mathrm{z}$ direction. Pre-optimization platform parameters are divided into 4 pairs, while the specific results of the analysis are shown in Fig. 5. Specifically, Fig. 5(a) shows that, stiffness and stroke exhibit opposite trend regarding changes in $R$ and $r$ values. The smaller the difference between $R$ and $r$, the more support the branch chain can provide along the $\mathrm{z}$ direction, in order to bear the load. Thus, variation rules of stiffness and travel can be summarized as: growing difference between $R$ and $r$ increases the stroke and reduces the stiffness. In Fig. 5(b), the maximum stiffness is achieved when $\alpha$ equals $\beta$. The effect of $\alpha$ on travel is less than that of $\beta$, whereas the smaller the $\beta$, the longer the travel applies. As shown in Fig. 5(c), the higher the length-diameter ratio of the compliant joint means longer travel and lower stiffness. Hence, it is significant to select an appropriate length-diameter ratio value of the compliant joint, in order to achieve the desired stiffness and travel. The scale of Fig. 5(c) demonstrates how a compliant joint is a crucial part, bearing great influence on the performance of the platform. Fig. 5(d) shows that, $D_{r}$ has no effect on travel and little effect on stiffness, whereas $L_{r}$ has little effect on travel but great effect on stiffness. Based on a suitable value of $D_{r}$, the effect of $D_{r}$ on stiffness and travel can be ignored, in the subsequent analysis.

Concluding, the parameters appear to influence stiffness and stroke of the platform in quite different ways. The ideal parameters, considering the desired stiffness and stroke, cannot be determined based directly on the figure. 

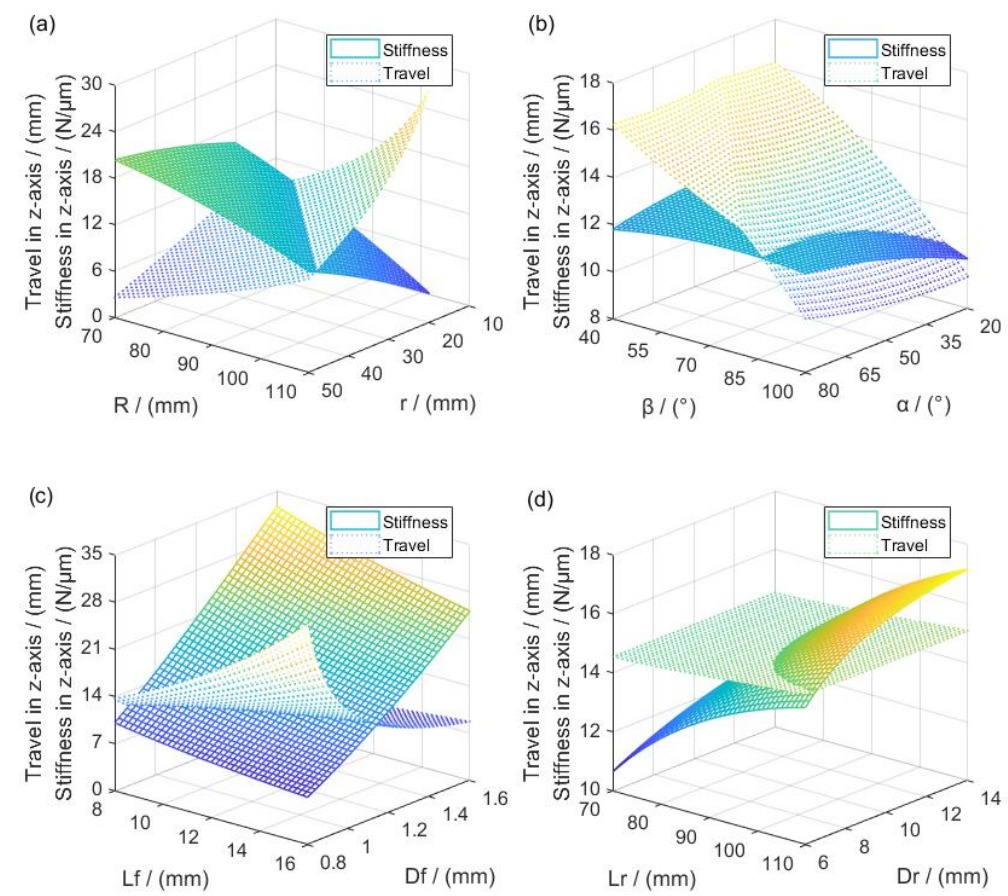

Fig. 5 The influence of structure parameters on travel and stiffness in the $\mathrm{z}$ direction.

\subsection{Parameter optimization}

Suitable values ought to be assigned to the stroke and stiffness of the platform, so as to meet application requirements. In practice, as is shown in Fig. 1, the load mainly acts along the $\mathrm{z}$ direction of the platform. Therefore, the stroke and stiffness in $z$ direction are taken as the optimization indexes, in this study. The desired stroke is set as $S_{z}=14 \mathrm{~mm}$ and the desired stiffness as $K_{z}=14 \mathrm{~N} / \mu \mathrm{m}$.

The parameters need to be rounded after optimization. However, even a slight change in the compliant joint diameter will have a great impact on platform performance. More precisely, due to the final rounding operation, the preferred parameter combination, as derived from optimization, can be invalid. Therefore, during the optimization, the diameter of compliant joint should be assigned a suitable constant value. In addition, to facilitate the installation of the fixture at the end of the optimized platform, the moving platform radius is also considered as a constant value. As the rigid rod diameter has little influence on the optimization, it is also set as a constant. In this study, the following are set: $D_{f}=1$ $\mathrm{mm}, r=25 \mathrm{~mm}, D_{r}=10 \mathrm{~mm}$. Therefore, the number of the structural parameters to be optimized reduces from 8 to 5 .

With the help of MATLAB, PSO is used to solve this multi-objective optimization problem. The multi-objective optimization is usually transformed into single-objective optimization, by changing the weight coefficient, as defined in the weighted sum method. In order to make the accuracy of the optimization results controllable, Gaussian distribution is for the first time used to transform the multi-objective optimization into a single-objective one:

$$
\operatorname{Fit}(X)=\sum_{n=1}^{N} \frac{w_{n}}{\sqrt{2 \pi} \sigma_{n}} \exp \left(-\frac{\left(X-\mu_{n}\right)^{2}}{2 \sigma_{n}^{2}}\right)
$$

where, Fit $(X)$ is the fitness function, $N$ is the number of the objectives to be 
optimized, $w_{n}, \sigma_{n}$, and $\mu_{n}$ are the weight coefficient, scale parameter, and location parameter of the $n^{\text {th }}$ optimization objective, respectively. In particular, the scale parameters determine the accuracy of the optimization results, whereas location parameters determine the value of the optimization objectives. Here, the multiobjective optimization is defined as:

$$
\operatorname{maximize} \text { Fit }=f\left(\sigma_{\max }\right)+f\left(k_{z}\right)
$$

subject to:

$$
\left\{\begin{array}{l}
70 \mathrm{~mm} \leq R \leq 110 \mathrm{~mm} \\
20^{\circ} \leq \alpha \leq 60^{\circ} \\
40^{\circ} \leq \beta \leq 80^{\circ} \\
7 \mathrm{~mm} \leq L_{f} \leq 19 \mathrm{~mm} \\
50 \mathrm{~mm} \leq L_{r} \leq 90 \mathrm{~mm}
\end{array}\right.
$$

In Eq. (4), $f\left(\sigma_{\max }\right)$ and $f\left(k_{\mathrm{z}}\right)$ refer to the probability density function of the maximum stress function at target point $\left(0,0, S_{z^{+}}\right)$and total stiffness function, respectively. The coefficients of $f\left(\sigma_{\max }\right)$ and $f\left(k_{\mathrm{z}}\right)$ are selected according to the expected accuracy of the optimization objectives. Here, $f\left(\sigma_{\max }\right)$ and $f\left(k_{\mathrm{z}}\right)$ are expressed as:

$$
\begin{aligned}
& f\left(\sigma_{\max }\right)=\frac{125}{\sqrt{2 \pi}} \exp \left(-\frac{\left(\sigma_{\max }-828\right)^{2}}{2}\right) \\
& f\left(k_{z}\right)=\frac{25}{0.2 \sqrt{2 \pi}} \exp \left(-\frac{\left(k_{z}-14\right)^{2}}{0.08}\right)
\end{aligned}
$$

The curves of $f\left(\sigma_{\max }\right)$ and $f\left(k_{\mathrm{z}}\right)$ are illustrated in Fig. 6 . When the fitness Fit approximates 100, the optimization objective is achieved. Fig. 7 shows the flowchart of the PSO. Fig. 8 shows 21 set of parameters of the optimized platform, where the red rectangle indicates the region of a pair. Among the parameters studied, $L_{f}$ shows the greatest influence on the stiffness and stroke of the platform, followed by $L_{r}, R, \beta$, and $\alpha$. The stroke of the platform in $\mathrm{x}$ and $\mathrm{y}$ directions is shown in Fig. 9, where it is evident that, longer compliant joint corresponds to wider workspace. In addition, the stroke of the platform in the $\mathrm{x}$ direction is larger than that in the y direction.

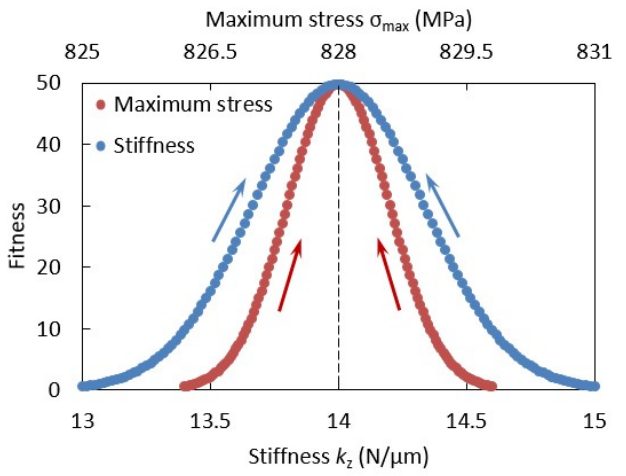

Fig. 6 Maximum stress and total stiffness. 


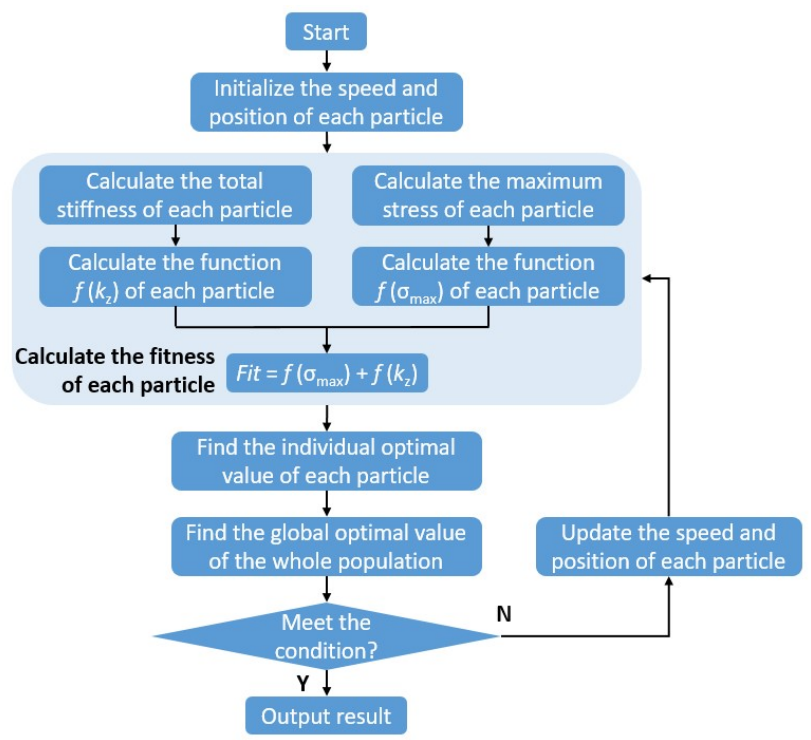

Fig. 7 Flowchart of the PSO.

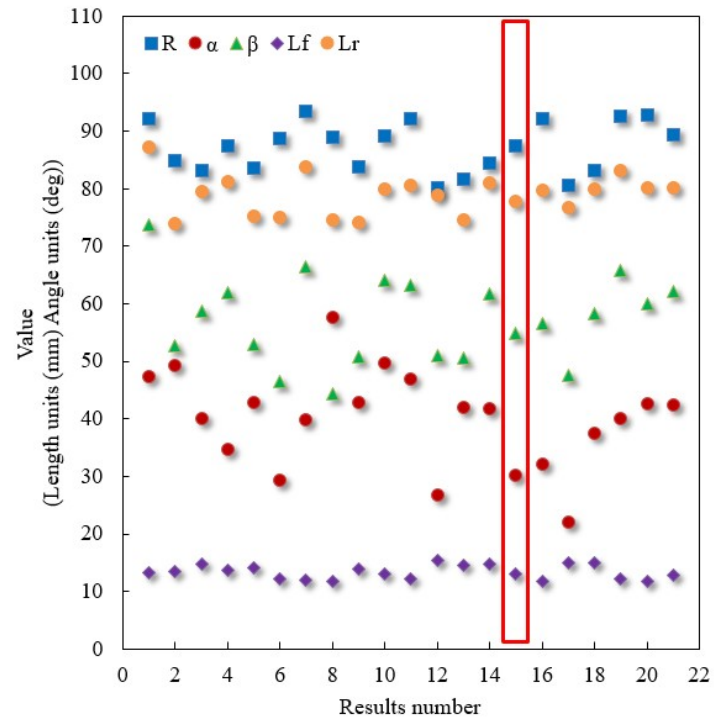

Fig. 8 Parameters of the optimized platform based on PSO.

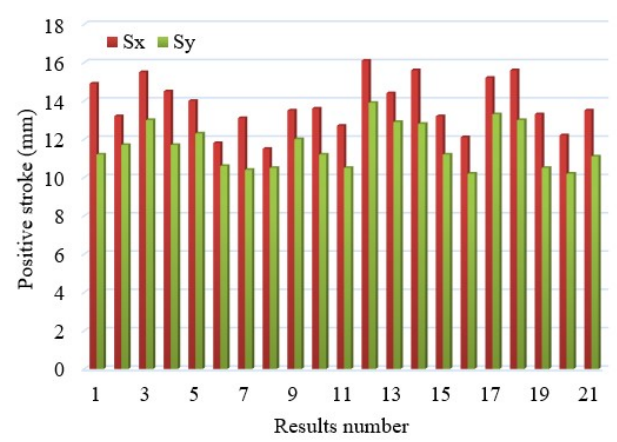

Fig. 9 The stroke of the platform in $\mathrm{x}$ and $\mathrm{y}$ directions based on Fig. 8 .

\section{Evaluation}

As mentioned above, the workspace and stiffness are the main performance indexes of the 6-PSS platform. In this section, after comparing results from NA, 
FEA, and experiment, the effectiveness of NA in obtaining the desired stiffness and workspace of the platform is verified. In addition, the effectiveness of PSO in multi-objective optimization is verified by comparing the platform performance before and after optimization.

\subsection{Workspace evaluation}

Fig. 3 shows that, the workspace of the platform is established, by employing the IKM to calculate the motion of the actuation units, when the moving platform moves to a target position. The maximum stress is used to determine whether the target point falls within the workspace of the platform. Therefore, the validity of the IKM and maximum stress criterion will determine the reliability of NA to obtain the workspace. According to the desired posture of the moving platform, the input posture of actuation units is obtained by IKM, while the output posture and the maximum stress of the platform are obtained by FEA. Next, the effectiveness of the IKM is validated by comparing the desired posture to the output posture. Similarly, the validity of the maximum stress criterion is confirmed by comparing the maximum stress value obtained by NA to the one derived from FEA. The parameters, as shown in the red rectangle in Fig. 8, are selected as the post-optimization structural parameters of the platform.

The stress cloud of FEA is shown in Fig. 10 and the specific simulation results are listed in Tables 4 and 5, where it is demonstrated that the output posture, as derived from FEA, matches well the desired posture. Insufficient mesh quality in FEA accounts for any slight deviations. Tables 4 and 5 demonstrate well the proximity of the maximum stress values, as derived by NA and by FEA, whereas any small difference may be due to nonlinear factors that affect the results of NA. In addition, the output posture of the moving platform is accurate, when the maximum stress is derived by NA; however, there is a slight deviation between the output and desired posture, when FEA is applied. Thus, the maximum stress of the platform, as calculated by FEA, surpasses what is calculated by NA. The comparison of the maximum stress values in Tables 4 and 5 indicates that the strokes in the $\mathrm{x}$ and $\mathrm{y}$ directions of the post-optimization platform are larger than the ones of the pre-optimization platform. In addition, according to Table 5, the stroke in $\mathrm{z}$ direction of the post-optimization platform is approximately equal to the desired stroke of $14 \mu \mathrm{m}$. Thus, it is concluded that the optimized results by PSO are reliable, whereas, considering all of the above, NA shows high precision and strong universal applicability in obtaining the maximum stress of the platform.
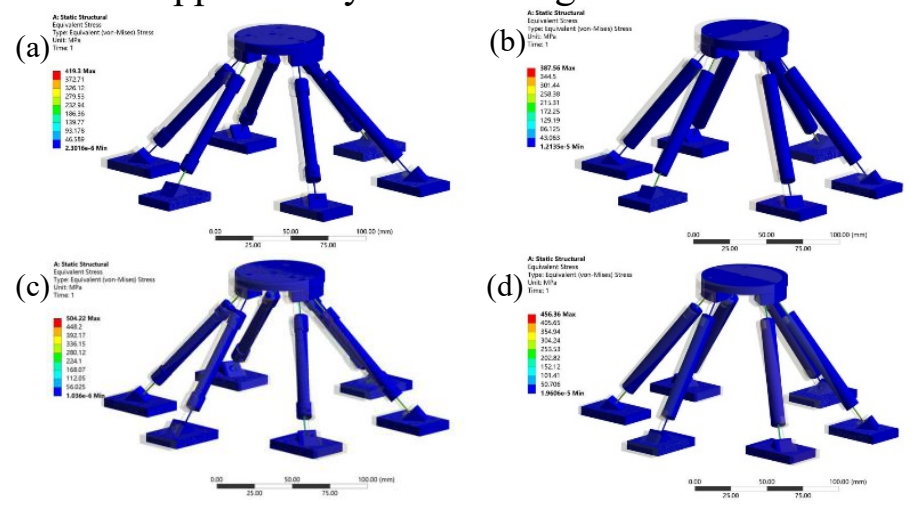

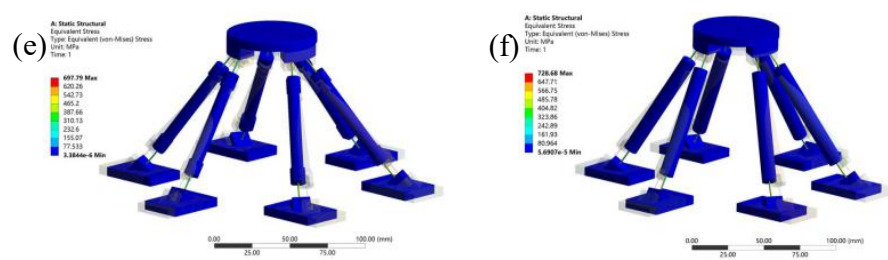

Fig. 10 The equivalent stress cloud at the desired posture of the platform. (a), (c), (e) refers to the equivalent stress at the desired posture of the pre-optimization platform; (b), (d), (f) refers to the equivalent stress at the desired posture of the post-optimization platform; the desired posture of (a), (b) is $(6,0,0,0,0,0)$; the desired posture of (c), (d) is $(0,6,0,0,0,0)$; the desired posture of (e), (f) is $(0,0,6,0,0,0)$.

Table 4 Results of the pre-optimization platform.

\begin{tabular}{|c|c|c|c|}
\hline \multirow{2}{*}{$\begin{array}{l}\text { Desired posture } \\
\text { (tx,ty,tz,rx,ry,rz) } \\
(\mathrm{mm}),(\mathrm{deg})\end{array}$} & \multirow{2}{*}{$\begin{array}{l}\text { Output posture by FEA } \\
(\mathrm{Tx}, \mathrm{Ty}, \mathrm{Tz}, \mathrm{Rx}, \mathrm{Ry}, \mathrm{Rz}) \\
(\mathrm{mm}),(\mathrm{deg})\end{array}$} & \multicolumn{2}{|c|}{$\begin{array}{c}\text { Maximum stress } \\
/(\mathrm{MPa})\end{array}$} \\
\hline & & FEA & NA \\
\hline$(6,0,0$, & $\begin{array}{c}(6.0002,0.0000,-0.0011, \\
0.0000,-0.0015,0.0000)\end{array}$ & 419.30 & 395.17 \\
\hline$(0,6,0,0,0,0)$ & $\begin{array}{l}(0.0000,5.9976,0.0000 \\
0.0029,0.0000,-0.0198)\end{array}$ & 504.22 & 483.32 \\
\hline$(0,0,6$ & $\begin{array}{c}(-0.0042,0.0000,5.9980 \\
0.0000,0.0092,0.0001)\end{array}$ & 97.79 & 71 \\
\hline
\end{tabular}

Table 5 Results of the post-optimization platform.

\begin{tabular}{cccc}
\hline $\begin{array}{c}\text { Desired posture } \\
\text { (tx,ty,tz,rx,ry,rz) }\end{array}$ & $\begin{array}{c}\text { Output posture by FEA } \\
(\mathrm{Tx}, \mathrm{Ty}, \mathrm{Tz}, \mathrm{Rx}, \mathrm{Ry}, \mathrm{Rz})\end{array}$ & \multicolumn{2}{c}{$\begin{array}{c}\text { Maximum stress } \\
(\mathrm{MPa})\end{array}$} \\
$(\mathrm{mm}),(\mathrm{deg})$ & $(\mathrm{mm}),(\mathrm{deg})$ & FEA & NA \\
\hline$(6,0,0,0,0,0)$ & $\begin{array}{c}(5.9949,0.0000,-0.0001, \\
\end{array}$ & 387.56 & 375.56 \\
& $0.0000,0.0175,0.0011)$ & & \\
$(0,6,0,0,0,0)$ & $(0.0000,5.9898,0.0000$, & 456.36 & 442.50 \\
& $0.0315,0.0000,0.0030)$ & & \\
$(0,0,6,0,0,0)$ & $(-0.0008,0.0000,5.9993$, & 728.68 & 709.64 \\
\hline
\end{tabular}

In order to obtain the workspace of the platform based on NA, the stroke of the platform in the directions of $\mathrm{x}, \mathrm{y}$, and $\mathrm{z}$ axis is first calculated. Next, a cuboid search space is formed by the stroke of the platform, in $\mathrm{x}, \mathrm{y}$, and $\mathrm{z}$ axes. Following, array points in the search space are considered as target points of the moving platform. The maximum stress of the platform is calculated, when the end of the moving platform overlaps the target points. By comparing the maximum stress to the allowable stress level, it is determined whether the target point falls within the workspace of the platform.

Fig. 11 shows the workspace of the pre-optimization platform, as derived by $\mathrm{NA}$ and FEA, as well as the projection of the workspace along the three coordinates axes. The different colors in the figure signify the variation of the maximum stress (MPa). Judging from the shape and coverage of the projection, the workspaces obtained by NA and FEA are almost identical. However, the computation demands and visibility of the results are quite different. Even after a high number of simulation runs, the disparity of the points in Fig. 11(b) makes it difficult to determine the exact shape of the workspace. On the contrary, the shape of the workspace shown in Fig. 11(a) is quite well defined; meaning that NA is faster and better than FEA in this task. 
(a)

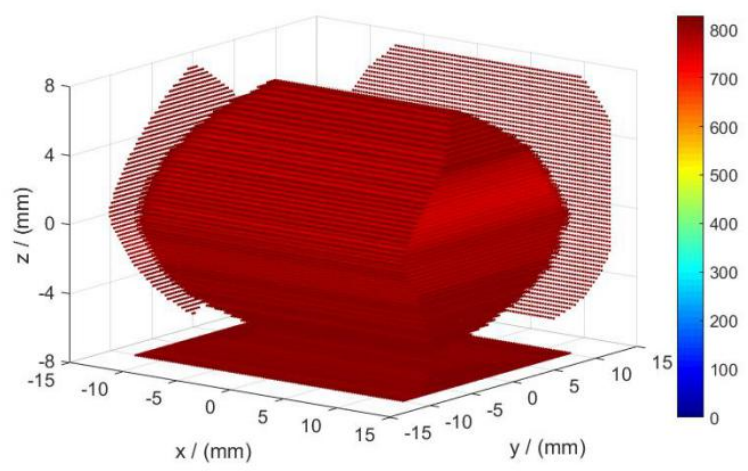

(b)

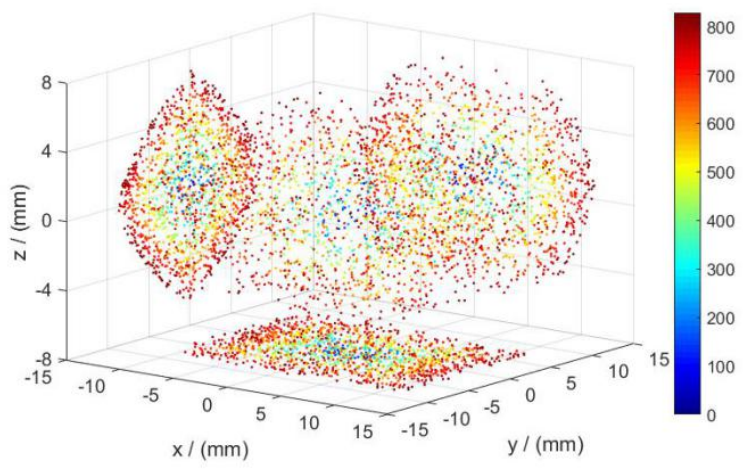

Fig. 11 Workspace of the platform. (a) Obtained by NA. (b) Obtained by FEA [17].

\subsection{Stiffness evaluation}

According to the FEA, at the initial state, the actuation units of the platform are fixed and a unit force is exerted along the z-axis direction, on the moving platform. Next, the displacement of the moving platform along the $z$-axis direction is measured. Then, according to Eq. (1), the stiffness in the z-axis direction is calculated. The results of FEA and NA are shown in Fig. 12. Due to its nonsymmetrical structure, the platform will deviate and deflect under the unit force, which means that the force making the platform move along z-axis direction is smaller than the unit force. Thus, the stiffness along the $\mathrm{z}$-axis, according to the FEA approach, is lower than the one calculated based on the NA method. Fig. 12 demonstrates that the stiffness of the post-optimization platform in the z-axis is significantly improved compared to that of the pre-optimization platform.

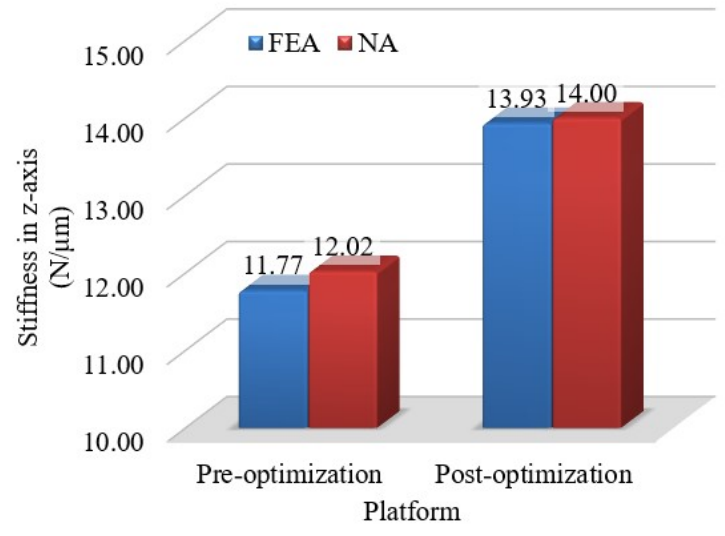

Fig. 12. The stiffness evaluation results.

According to Eq. (1), considering that load and displacement are the critical 
factors in stiffness testing, their accuracy will directly affect the reliability of the results. In the FEA approach, the reliability of the test results is guaranteed by accurately setting the boundary conditions. Contrary to the experimental method, the boundary conditions in FEA are idealized facilitating the load assignment and displacement measurement. In the previous experiment, the weight exerts directly on the platform, as the load and the displacement of the platform are obtained by the displacement sensors. However, the moving platform may move along other directions, making the test results inaccurate, as it is hard to ensure that the gravity vector of the load passes through the center of the moving platform. In addition, the measurement accuracy of the displacement sensor is restrained. Therefore, the accuracy of the static stiffness, when measured by experiment, needs to be further improved.

Hence, a novel method to measure the static stiffness of the platform is proposed. The load acting on the center of the moving platform is provided by the gravity of weights hanging by a rope, wrapped around two pulleys. The displacement of the moving platform is measured by a laser interferometer. The advantages of the method are about the load exerting directly on the center of the moving platform, being adjustable and along the Z-axis, while the derived measurement accuracy of displacement is high. The experimental setup and schematic diagram are depicted in Fig. 13.

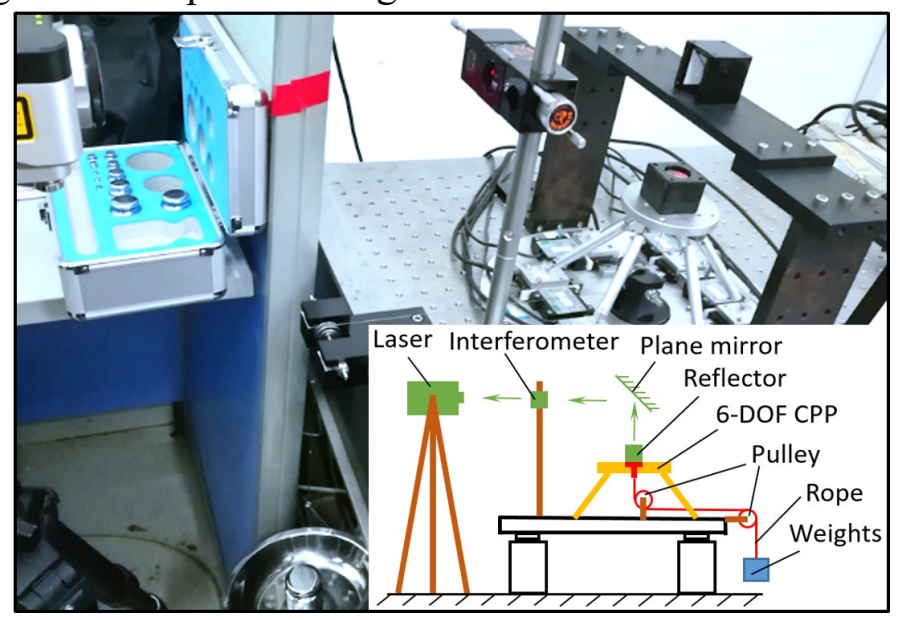

Fig. 13. The experimental setup and schematic diagram.

Similar to the FEA method, all actuation units are fixed at the initial state of the platform. Next, a load exerts on the center of the moving platform, causing a displacement along the $\mathrm{z}$ direction, which is measured by laser interferometer. The tested load values, along with the corresponding platform stiffness, are listed in Table 6, while the final experimental result is actually the average of 5 test iterations for every load. According to the Table 6 data, the stiffness along $\mathrm{z}$ direction shows some fluctuation, around the value of $11.56 \mathrm{~N} / \mu \mathrm{m}$, which is very close to the result derived by FEA and NA, whereas the slight deviation may be caused by machining and installation error. Regarding the compliant joints, even a slight error during machining or installation will have a significant impact on the performance of the platform. However, in general terms, the proposed NA is effective for the calculation of the stiffness of CPP.

Table 6 The experimental results.

\begin{tabular}{ccc}
\hline $\begin{array}{c}\text { Load } \\
(\mathrm{N})\end{array}$ & $\begin{array}{c}\text { Displacement } \\
(\mu \mathrm{m})\end{array}$ & $\begin{array}{c}\text { Stiffness in z-axis } \\
(\mathrm{N} / \mu \mathrm{m})\end{array}$ \\
\hline 5 & 0.439 & 11.39
\end{tabular}




\begin{tabular}{lll}
10 & 0.865 & 11.51 \\
15 & 1.268 & 11.65 \\
20 & 1.696 & 11.55 \\
25 & 2.126 & 11.67 \\
30 & 2.547 & 11.57 \\
\hline
\end{tabular}

\section{Conclusion}

Stiffness and workspace reflect the manipulation performance of a precision mechanism. In this paper, a numerical optimization method is presented for CPP, to achieve both desired stiffness and workspace, at the same time. The numerical model reveals the relationships between structural parameters, desired stiffness, and workspace, as the stiffness model and the workspace model are established based on elastokinematic analysis and allowable stress level of compliant joint, respectively. In addition, convergence precision is guaranteed by the use of Gaussian function for the transformation of the multi-objective optimization into a single-objective one. In order to obtain the desired stiffness and workspace of CPP, five structural parameters are optimized by PSO, significantly improving its stiffness and workspace.

\section{Declarations}

Funding: This work was supported in part by the National Natural Science Foundation of China under Grant 51975590.

Conflict of interest: The authors declare that they have no conflict of interest.

Availability of data and material: The datasets generated during and/or analysed during the current study are available from the corresponding author on reasonable request.

Code availability: Not applicable.

Authors' contributions: Not applicable.

Ethics approval: Not applicable.

Consent to participate: Not applicable.

Consent for publication: The authors consent to the publication of the paper.

\section{References}

[1] Fiore, E., Giberti, H., Ferrari, D.: Dynamics Modeling and Accuracy Evaluation of a 6-DoF Hexaslide Robot. Nonlinear Dynamics. 1, 473-479 (2017).https://doi.org/10.1007/978-3-319-15221-9 41. 
[2] Yun, Y., Li, Y.: Design and analysis of a novel 6-DOF redundant actuated parallel robot with compliant hinges for high precision positioning. Nonlinear Dynamics. 61(4), 829-845 (2010).https://doi.org/10.1007/s11071010-9690-x.

[3] Liu, M., Zhang, X., Fatikow, S.: Design and analysis of a multi-notched flexure hinge for compliant mechanisms. Precision Engineering. 48, 292-304 (2017).https://doi.org/10.1016/j.precisioneng.2016.12.012.

[4] Du, Z., Shi, R., Dong, W.: A Piezo-Actuated High-Precision Flexible Parallel Pointing Mechanism: Conceptual Design, Development, and Experiments. IEEE Transactions on Robotics. 30(1), 131-137 (2014).https://doi.org/10.1109/tro.2013.2288800.

[5] Dan, W., Rui, F.: Design and nonlinear analysis of a 6-DOF compliant parallel manipulator with spatial beam flexure hinges. Precision Engineering. 45, 365-373 (2016).https://doi.org/10.1016/j.precisioneng.2016.03.013.

[6] Huang, G., Guo, S., Zhang, D., Qu, H., Tang, H.: Kinematic analysis and multi-objective optimization of a new reconfigurable parallel mechanism with high stiffness. $\quad$ Robotica. 36(2), 187-203 (2017).https://doi.org/10.1017/s0263574717000236.

[7] Qiang, H., Wang, L., Ding, J., Zhang, L.: Multiobjective Optimization of 6DOF Parallel Manipulator for Desired Total Orientation Workspace. Mathematical Problems in Engineering. 2019, 1-10 (2019).https://doi.org/10.1155/2019/5353825.

[8] Dong, W., Du, Z. J., Sun, L. N.: Stiffness influence atlases of a novel flexure hinge-based parallel mechanism with large workspace. Intelligent Robots and Systems, Alberta, Canada, 2005.https://ieeexplore.ieee.org/document/1545078/.

[9] Chi, Z., Zhang, D., Xia, L., Gao, Z.: Multi-objective optimization of stiffness and workspace for a parallel kinematic machine. International Journal of Mechanics and Materials in Design. 9(3), 281-293 (2013).https://doi.org/10.1007/s10999-013-9219-9.

[10] Rastgar, H., Naeimi, H. R., Agheli, M.: Characterization, validation, and stability analysis of maximized reachable workspace of radially symmetric hexapod machines. Mechanism and Machine Theory. 137, 315-335 (2019).https://doi.org/10.1016/j.mechmachtheory.2019.01.033. 
[11] Zhu, D., Zhan, W.: Topology optimization of a 6-DOF spatial compliant mechanism based on Stewart propotype platform. Acta Mechanica Sinica. 35(5), 1044-1059 (2019).https://doi.org/10.1007/s10409-019-00877-8.

[12] Ganesh, S. S., Koteswara Rao, A. B., Darvekar, S.: Multi-objective optimization of a 3-DOF translational parallel kinematic machine. Journal of Mechanical Science and Technology. 27(12), 3797-3804 (2013).https://doi.org/10.1007/s12206-013-0957-2.

[13] Russo, M., Herrero, S., Altuzarra, O., Ceccarelli, M.: Kinematic analysis and multi-objective optimization of a 3-UPR parallel mechanism for a robotic leg. Mechanism and Machine Theory. 120, 192-202 (2018).https://doi.org/10.1016/j.mechmachtheory.2017.10.004.

[14] Kelaiaia, R., Zaatri, A., Company, O.: Multiobjective Optimization of 6-dof UPS Parallel Manipulators. Advanced Robotics. 26(16), 1885-1913 (2012).https://doi.org/10.1080/01691864.2012.703168.

[15] Shirazi, A. R., Fakhrabadi, M. M. S., Ghanbari, A.: Optimal Design of a 6DOF Parallel Manipulator Using Particle Swarm Optimization. Advanced Robotics. 26(13), $1419-1441$ (2012).https://doi.org/10.1080/01691864.2012.690187.

[16] Shang, J., Tian, Y., Li, Z., Wang, F., Cai, K.: A novel voice coil motordriven compliant micropositioning stage based on flexure mechanism. Rev Sci Instrum. 86(9), 095001 (2015).https://doi.org/10.1063/1.4929867.

[17] Xu, H., Zhou, H., Tan, S., Duan, J.-A., Hou, F.: A Six-Degree-of-Freedom Compliant Parallel Platform for Optoelectronic Packaging. IEEE Transactions on Industrial Electronics. 68(11), 11178-11187 (2021).https://doi.org/10.1109/tie.2020.3036225. 